\title{
Clinical Utility Gene Card For: GALNT3 defective congenital disorder of glycosylation
}

\author{
Jaak Jaeken ${ }^{1} \cdot$ Dirk J. Lefeber ${ }^{2} \cdot$ Gert Matthijs $^{3}$
}

Received: 1 March 2017 / Revised: 21 July 2017 / Accepted: 23 August 2017 / Published online: 23 April 2018

(c) European Society of Human Genetics 2018

\section{Disease characteristics}

\subsection{Name of the disease (synonyms)}

Deficiency of UDP- $N$-acetyl- $\alpha$-D-galactosamine:polypeptide $N$-acetylgalactosaminyltransferase 3 , deficiency of GalNAc transferase 3, deficiency of GalNAcT3, GALNT3 deficiency, GALNT3-CDG, familial hyperphosphatemic tumoral calcinosis, HFTC, hyperostosis-hyperphosphatemia syndrome, lipocalcinogranulomatosis, familial Teutschlaender disease.

\subsection{OMIM\# of the disease}

211900.

\subsection{Name of the analysed gene or DNA/chromosome segments}

GALNT3.

\subsection{OMIM\# of the gene}

601756.

Review of the analytical and clinical validity, as well as of the clinical utility of DNA-based testing for mutations in GALNT3 in diagnostic, predictive and prenatal settings, and for risk assessment in relatives.

Jaak Jaeken

jaak.jaeken@kuleuven.be

1 Centre for Metabolic Disease, University Hospital Gasthuisberg, KU Leuven, Leuven, Belgium

2 Department of Neurology, Translational Metabolic Laboratory, Radboudumc, Nijmegen, The Netherlands

3 Department of Human Genetics, KU Leuven, Leuven, Belgium

\subsection{Mutational spectrum}

At least thirty-five variants have been reported: eleven missense variants, twenty-two nonsense variants, and two splice variants (www.lovd.nl/GALNT3). There are no obvious hotspot variants. The standard reference sequence indicating reported variants (ENSG00000115339) and a reference for exon numbering (ENST00000392701) can be found at http://www.ensembl.org.

\subsection{Analytical methods}

Sanger sequencing of the 13 coding exons and flanking intronic sequences of the GALNT3 gene (NCBI reference sequence: NM_004482.3).

\subsection{Analytical validation}

Sanger sequencing identifies variants in $>99 \%$ of patients. Deep intronic variants, large deletions, and duplications would not be detected using this approach. Novel variants with uncertain effect on function are of course possible.

\subsection{Estimated frequency of the disease (Incidence at birth ("birth prevalence") or population prevalence)}

If known to be variable between ethnic groups, please report:

At least sixty-six genetically confirmed patients (from 42 families) have been reported [1-3]. The frequency and the prevalence of the disease are not known.

\subsection{Diagnostic setting}

Yes No

A. (Differential) diagnostics $\quad \square$ 
Table (continued)

\begin{tabular}{lcc} 
B. Predictive testing & $\bigotimes$ & \\
C. Risk assessment in relatives & $\bigotimes$ & $\square$ \\
D. Prenatal & $\square$ & $\square$ \\
\hline
\end{tabular}

\section{Comment:}

Deficiency of $\mathrm{N}$-acetylgalactosaminyltransferase 3 is an autosomal recessive disorder of $\mathrm{O}$-glycosylation, first reported in 2004 [4]. GalNAc transferase 3 belongs to a large family of at least 23 Golgi-associated enzymes and transfers GalNAc from the sugar donor UDP-GalNAc to serine and threonine residues thus initiating mucin-type $\mathrm{O}-$ glycosylation. GALNT3-CDG belongs to the congenital disorders of glycosylation (CDG), a large group of genetic defects in protein and lipid glycosylation. Most CDG are multisystem disorders with prominent neurological involvement. More than 100 CDG have been described. Subtype identification is challenging due to the large clinical and genetic heterogeneity. There are protein glycosylation defects in N-glycosylation and O-glycosylation.

GALNT3-CDG is the most prevalent genetic cause of familial tumoral calcinosis and is a hyperphosphatemic calcinosis. It is characterized by the presence of ectopic calcifications in soft tissues around major joints (shoulders, elbows, hips, knees etc.). In some patients, there are also vascular calcifications, angioid streaks of the retina, dental abnormalities, and/or testicular microlithiasis. This can lead to intolerable pain, skin ulcerations, and secondary skin and bone infections. Another presentation is the hyperostosishyperphosphatemia syndrome characterized by recurrent long bone lesions [5]. Both presentations represent a continuous spectrum of the same disease and can occur in the same patient. Clinical manifestations vary even within families, from asymptomatic to large, disabling calcifications. There is no phenotype-genotype correlation. The disease is predominantly present in black African and Middle-Eastern patients. A hallmark biochemical feature is persistent hyperphosphatemia with elevated or inappropriately normal 1,25 hydroxy-vitamin D. Serum calcium and parathyroid hormone levels are usually normal. Imaging tests of the calcifications can resemble those of several other disorders including primary hyperparathyroidism, vitamin D poisoning, dermatomyositis, and neoplastic conditions. The hyperphosphatemia is due to increased renal phosphate reabsorption secondary to decreased O-glycosylation of FGF23 by the deficient GALNT3 protein. By glycosylating FGF23, GALNT3 protects this phosphaturic protein from proteolytic processing. Intact serum FGF23 levels are thus decreased in GALNT3-CDG. Current screening tests for defects in O-glycosylation (mainly apo C-III isoelectrofocusing) show normal results The diagnosis of GALNT3-CDG is based on the clinical acumen of the physician and confirmed by mutation analysis of GALNT3. The identification of the pathogenic variant will permit heterozygote detection in the family, and prenatal diagnosis. Recent reviews provide more details [1, 6].

\section{Test characteristics}

\begin{tabular}{|c|c|c|}
\hline $\begin{array}{l}\text { Genotype or } \\
\text { disease }\end{array}$ & A: True positives & $\begin{array}{l}\mathrm{C} \text { : False } \\
\text { negatives }\end{array}$ \\
\hline Present Absent & B: False positives & $\begin{array}{l}\text { D: True } \\
\text { negatives }\end{array}$ \\
\hline
\end{tabular}

Test

$\begin{array}{llll}\text { Postive A } & \text { B } & \text { Sensitivity: } & \mathrm{A} /(\mathrm{A}+\mathrm{C}) \\ & & \begin{array}{l}\text { Specificity: } \\ \text { Negative C }\end{array} & \mathrm{D} /(\mathrm{D}+\mathrm{B}) \\ & & \begin{array}{l}\text { Positive predic- } \\ \text { tive value: }\end{array} & \mathrm{A} /(\mathrm{A}+\mathrm{B}) \\ & \begin{array}{l}\text { Negative predic- } \\ \text { tive value: }\end{array} & \mathrm{D} /(\mathrm{C}+\mathrm{D}) \\ & & \end{array}$

\subsection{Analytical sensitivity}

(proportion of positive tests if the genotype is present)

Not applicable since there is no test available.

\subsection{Analytical specificity}

(proportion of negative tests if the genotype is not present) See 2.1.

\subsection{Clinical sensitivity}

(proportion of positive tests if the disease is present)

The clinical sensitivity can be dependent on variable factors such as age or family history. In such cases a general statement should be given, even if a quantification can only be made case by case.

See 2.1 .

\subsection{Clinical specificity}

(proportion of negative tests if the disease is not present)

The clinical specificity can be dependent on variable factors such as age or family history. In such cases a general statement should be given, even if a quantification can only be made case by case.

See 2.1. 


\subsection{Positive clinical predictive value}

(life time risk to develop the disease if the test is positive)

See 2.1 .

\subsection{Negative clinical predictive value}

(Probability not to develop the disease if the test is negative)

Assume an increased risk based on family history for a non-affected person. Allelic and locus heterogeneity may need to be considered.

Index case in that family had been tested:

See 2.1 .

Index case in that family had not been tested:

See 2.1 .

\section{Clinical utility}

\section{1 (Differential) diagnostics: the tested person is clinically affected}

(To be answered if in 1.9 "A" was marked)

\subsubsection{Can a diagnosis be made other than through a} genetic test?

\begin{tabular}{lll}
\hline No. & $\bigotimes($ Continue with 3.1.4) \\
Yes & Clinically & \\
& Imaging & $\square$ \\
& Endoscopy & $\square$ \\
& Biochemistry & \\
& Electrophysiology & $\square$ \\
& Other (please describe) \\
\hline
\end{tabular}

\subsubsection{Describe the burden of alternative diagnostic} methods to the patient

Not applicable.

3.1.3 How is the cost effectiveness of alternative diagnostic methods to be judged?

Not applicable.
3.1.4 Will disease management be influenced by the result of a genetic test?

No.

Yes. $\otimes$

Therapy (please
describe)

Treatment of GALNT3-CDG is only symptomatic and has a pharmacological and a surgical component. Low phosphate diet, phosphate binders and phosphaturia-inducing therapies have been attempted with variable response. Since this is a rare disease, no randomized clinical trials have been performed. As to surgical treatment, some patients showed complete resolution of calcinosis lesions after removal while others required multiple repeated surgeries due to lesion recurrence [6]

Prognosis (please Molecular testing is essential for describe) confirmation of the diagnosis and genetic counseling of the families concerned

Management GALNT3-CDG is a multi-system (please describe) disease, mainly involving skin, bones, teeth and eyes. Follow-up by a multidisciplinary team is thus important

\subsection{Predictive setting: the tested person is clinically unaffected but carries an increased risk based on family history}

(To be answered if in 1.9 "B" was marked)

3.2.1 Will the result of a genetic test influence lifestyle and prevention?

If the test result is positive (please describe):

Not applicable.

If the test result is negative (please describe):

Not applicable.

3.2.2 Which options in view of lifestyle and prevention does a person at-risk have if no genetic test has been done (please describe)?

Not applicable. 
3.3 Genetic risk assessment in family members of a diseased person

(To be answered if in 1.9 "C" was marked)

3.3.1 Does the result of a genetic test resolve the genetic situation in that family?

Usually yes, by testing the potential heterozygous persons (carriers) in the family.

3.3.2 Can a genetic test in the index patient save genetic or other tests in family members?

No.

3.3.3 Does a positive genetic test result in the index patient enable a predictive test in a family member?

Not applicable.

\subsection{Prenatal diagnosis}

(To be answered if in 1.9 "D" was marked)

\subsubsection{Does a positive genetic test result in the index patient enable a prenatal diagnosis?}

Yes. Prenatal diagnosis should be performed by molecular analysis.

\section{If applicable, further consequences of testing}

Please assume that the result of a genetic test has no immediate medical consequences. Is there any evidence that a genetic test is nevertheless useful for the patient or his/her relatives? (Please describe).

Knowledge of the diagnosis will stop unnecessary further investigations, and will help patients and parents of affected children in the process of accepting the disease although no curative treatment is available.

Acknowledgements This work was supported by EuroGentest2 (Unit 2: "Genetic testing as part of health care"), a Coordination Action under FP7 (Grant Agreement Number 261469) and the European Society of Human Genetics.

\section{Compliance with ethical standards}

Conflict of interest The authors declare that they have no conflict of interest.

\section{References}

1. Rafaelsen S, Johansson S, Ræder H, Bjerkness R. Long-term clinical outcome and phenotypic variability in hyperphosphatemic familial tumoral calcinosis and hyperphosphatemic hyperostosis syndrome caused by a novel GALNT3 mutation; case report and review of the literature. BMC Genet. 2014;15:98.

2. Ramnitz MS, Gourh P, Goldbach-Mansky R, et al. Phenotypic and genotypic characterization and treatment of a cohort with familial tumoral calcinosis/hyperostosis-hyperphosphatemia syndrome. J Bone Miner Res. 2016;31:1845-54.

3. Sun L, Zhao L, Du L, et al. Identification of two novel mutations in the GALNT3 gene in a Chinese family with hyperphosphatemic familial tumoral calcinosis. Bone Res. 2016;4: https://doi.org/10. 1038/boneres.2016.38.

4. Topaz O, Shurman DI, Bergman R, et al. Mutations in GALNT3, encoding a protein involved in O-linked glycosylation, cause familial tumoral calcinosis. Nat Genet. 2004;36:579-81.

5. Frishberg Y, Topaz O, Bergman R., et al. Identification of a recurrent mutation in GALNT3 demonstrates that hyperostosishyperphosphatemia syndrome and familial tumoral calcinosis are allelic disorders. J Mol Med. 2005;83:33-8.

6. Folsom LJ, Imel EA. Hyperphosphatemic familial tumoral calcinosis: genetic models of deficient FGF23 action. Curr Osteoporos Rep. 2015;13:78-87. 